\title{
APPROXIMATE CONTINUITY AND LINEAR APPROXIMATE CONTINUITY
}

\author{
CASPER GOFFMAN
}

(Communicated by Andrew M. Bruckner)

\begin{abstract}
A contrast between approximated continuity and continuity is obtained similar to the classical one for derivatives.
\end{abstract}

One of the foremost achievements in the theory of real functions is the work of Stepanoff on approximate differentiation of functions of several variables. It was known early that functions of two variables could have partial derivatives almost everywhere but be nowhere differentiable. Such an example, absolutely continuous in the sense of Tonelli, and accordingly having partial derivatives almost everywhere, was given by Stepanoff [1], which had a total differential nowhere. Then Stepanoff proved the remarkable fact that if $f$ is a measurable real function of $n$ variables, then $f$ has an approximate total differential almost everywhere if and only if $f$ has approximate partial derivatives almost everywhere [2].

We note that a similar development holds for continuity and linear continuity, ordinary and approximate.

We define linear continuity and linear approximate continuity. These notions are not coordinate invariant but are defined relative to a given system of rectangular coordinates in euclidean $n$-space. A real function is linearly continuous if its restriction to each line parallel to a coordinate axis is a continuous function relative to the line. A similar definition holds for linear approximate continuity.

Let $f$ be a measurable real function of $n$ variables. It is well known that $f$ is approximately continuous almost everywhere. In $\S 2$ we shall note that if $f$ is measurable it is also linearly approximately continuous almost everywhere.

2

It is known that if $S$ is measurable then the linear density of $S$ is 1 in every coordinate direction at almost every point in $S$ [3]. This implies that if $f$ is measurable then $f$ is linearly approximately continuous almost everywhere.

Theorem 1. If $f$ is measurable then $f$ is approximately continuous almost everywhere if and only if $f$ is linearly approximately continuous almost everywhere.

Received by the editors July 17, 1990 and, in revised form, March 19, 1992.

1991 Mathematics Subject Classification. Primary 26B05. 
In contrast, we show that a function $f$ may be linearly continuous everywhere and be discontinuous on a set of positive measure. Let $n=2$. We start with a model function. Let $s$ be a line segment starting at $\left(x_{0}, y_{0}\right)$ at an angle $\pi / 4$ with the positive $x$-axis. Let $\left\{\left(x_{n}, y_{n}\right)\right\}$ be a sequence on $s$ converging to $\left(x_{0}, y_{0}\right)$. Construct a linearly continuous function $f$ which is 0 on the horizontal and vertical lines through $\left(x_{0}, y_{0}\right)$ and whose value is 1 at each $\left(x_{n}, y_{n}\right)$. Then $f$ is discontinuous, with oscillation 1 , at $\left(x_{0}, y_{0}\right)$.

Let $Q=[0,1] \times[0,1]$, and let $S \subset Q$, with $S=A \times B$, where $A$ and $B$ are Cantor type sets of positive measure. We define a linearly continuous $f$ which is 0 on $S$ and has oscillation 1 at every point of $S$. The construction is made as follows.

Let $I_{n}, J_{m}$ be components of the complements of $A$ and $B$, respectively. Let $\left(x_{n}, y_{m}\right)$ be the lower left-hand end point of $I_{n} \times J_{m}$, and let the linearly continuous $f$ be 1 on a sequence in $I_{n} \times J_{m}$ converging to $\left(x_{n}, y_{m}\right), n, m=$ $1,2, \ldots$. The function $f$ is linearly continuous but is discontinuous at every point in $S$. (The set $S$ is an example of a sectionally zero-dimensional set, a type of set introduced in [4] in order to extend homeomorphisms in dimension greater than 2.)

\section{4}

We note that, for $n=2$, linearly continuous functions are of Baire class 1 .

Suppose $f$ is linearly continuous on $Q=[0,1] \times[0,1]$. For each $m$, let

$$
f_{m}(k / m, y)=f(k / m, y) \quad \text { for } k=0,1, \ldots, m, y \in[0,1] .
$$

For $k / m \leq x \leq(k+1) / m$, extend $f_{m}$ linearly from $(k / m, f(k / m))$ to $((k+1) / m, f((k+1) / m))$. Then each $f_{m}$ is continuous and $\left\{f_{m}\right\}$ converges uniformly to $f$ in $x$ for every $y$.

A corollary is that a linearly continuous function cannot be discontinuous everywhere.

We cite the following questions.

(i) In connection with Theorem 1 , is it true that every real $f$ which is linearly approximately continuous almost everywhere is measurable?

(ii) What are the Baire properties of linearly continuous functions for $n>$ 2 ?

\section{ACKNOWLEDGMENT}

The writer wishes to thank the referee for his pertinent remarks regarding an earlier version of this note.

\section{REFERENCES}

1. W. Stepanoff, Uber totale Differenzierbarkeit, Math. Ann. 90 (1923), 318-320.

2. _ Sur les conditions de l'existence de la differentielle total, Math. Soc. Math. Moscow 32 (1925), 511-525.

3. S. Saks, Theory of the integral, Warsaw, 1937, p. 130.

4. C. Goffman, One-one measurable transformations, Acta Math. 89 (1953), 261-278.

Department of Mathematics, Purdue University, West lafayette, Indiana 47907 